OPEN ACCESS

Edited by:

Nicole Thielens,

UMR5075 Institut de Biologie

Structurale (IBS), France

Reviewed by:

Rostyslav Bilyy,

Danylo Halytsky Lviv National Medical

University, Ukraine

Bernd Lepenies,

University of Veterinary Medicine

Hanover, Germany

*Correspondence:

Sebastian P. Galuska galuska.sebastian@

fbn-dummerstorf.de

Specialty section: This article was submitted to

Molecular Innate Immunity,

a section of the journal

Frontiers in Immunology

Received: 14 February 2019 Accepted: 15 April 2019

Published: 07 May 2019

Citation:

Bornhöfft KF and Galuska SP (2019)

Glycans as Modulators for the

Formation and Functional Properties

of Neutrophil Extracellular Traps: Used by the Forces of Good and Evil.

Front. Immunol. 10:959.

doi: 10.3389/fimmu.2019.00959

\section{Glycans as Modulators for the Formation and Functional Properties of Neutrophil Extracellular Traps: Used by the Forces of Good and Evil}

\author{
Kim F. Bornhöfft and Sebastian P. Galuska* \\ Institute of Reproductive Biology, Leibniz Institute for Farm Animal Biology, Dummerstorf, Germany
}

A very common mechanism to trap pathogens is the release of DNA. Like flies in a spider's web, pathogens are enclosed in a sticky chromatin meshwork. Interestingly, plants already use this mechanism to catch bacteria. In mammals, especially neutrophils release their DNA to prevent an invasion of bacteria. These neutrophil extracellular traps (NETs) are equipped with antimicrobial molecules, including, for instance, histones, antimicrobial peptides, lactoferrin, and neutrophil elastase. Thus, in a defined area, pathogens and toxic molecules are directly adjacent. However, several of these antimicrobial substances are also cytotoxic for endogenous cells. It is, therefore, not surprising that distinct control mechanisms exist to prevent an exaggerated NETosis. Nevertheless, despite these endogenous control instruments, an extraordinary NET release is characteristic for several pathologies. Consequently, NETs are a novel target for developing therapeutic strategies. In this review, we summarize the roles of glycans in the biology of NETs; on the one hand, we focus on the glycan-dependent strategies of endogenous cells to control NET formation or to inactivate its cytotoxic effects, and, on the other hand, the "sweet" tricks of pathogens to inhibit the release of NETs or to prevent NET-mediated killing mechanisms are examined. Understanding both, the forces of good and evil, allows the development of novel glycan-based approaches to combat the harmful side of NETs during distinct pathologies.

Keywords: NETosis, Siglecs, polysialic acid, histones, glycosaminoglycans

\section{INTRODUCTION}

Neutrophil granulocytes possess a panel of different mechanisms to combat invading pathogens. As first line of defense neutrophils can combat pathogens by phagocytosing them, by releasing antimicrobial peptides or reactive oxygen species (ROS) and by the release of neutrophil extracellular traps (NETs), a process first described in 2004 by Brinkmann et al. $(1,2)$. NET release can be induced by bacteria, viruses, fungi or non-physiological stimuli, such as ionophores and phorbol-myristate acetate (PMA) $(3,4)$. It seems to be that different pathways can be used to induce the formation of NET and even more than 13 years after the first description of NETs several mechanisms are controversially discussed and numerous open questions still need to be answered [excellently summarized in Boeltz et al. (5)]. What we know is that NET consists of a meshwork of decondensed DNA fibers, cytotoxic histones, and antimicrobial peptides and has the ability to catch and render invading pathogens harmless $(3,5,6)$. Since NET contains several biomolecules, 
which are also cytotoxic for endogenous cells, in addition to the desired antimicrobial effects, NETs are associated with numerous pathologies (7-16). Therefore, an exaggerated release of NET has to be prevented, leading to the necessity of control mechanisms to regulate the formation of NETs.

Within the last few years, interest in glycans and how they modulate different functions has increased immensely (1719). Since all of our cells are surrounded by a glycocalyx consisting of highly glycosylated proteins and lipids, it is obvious that glycan-dependent processes occur frequently (19, 20). Indeed, glycans have essential roles within various biological processes, such as cell proliferation, cell differentiation, the development of organs, and within the immune system $(19,21)$. In the field of immunology, glycans drive diverse mechanisms, ranging from the discrimination of the self and non-self, using for instance sialic acid-binding immunoglobulin-like lectins (Siglecs), to glycosaminoglycan (GAG)-mediated chemokine presentation (22).

GAGs are a number of long, unbranched polysaccharides composed of repeating disaccharide units, whereby, the repeating disaccharides consist of uronic acid or galactose and an amino sugar, which can be additionally modified. Prominent members of the GAG family are: hyaluronic acid, heparin/heparan sulfate, chondroitin sulfate/dermatan sulfate, and keratin sulfate (23). Besides their role in chemokine presentation, GAGs are also involved in several other biological processes, like cell signaling, angiogenesis $(24)$, metastasis, tumor progression $(25,26)$ and coagulation $(27,28)$.

In addition to GAGs, sialylated glycans play an important role during immunological events. In mammals, glycans are frequently terminated by these sugar residues (29). Sialic acids are negatively charged and modulate both immunological processes and organ development (30-33). Sialylated structures can be recognized by Siglecs (34-36), which are transmembrane receptors expressed in different cells of vertebrates that can mainly either inhibit or activate the immune response (35, 37-39). Although Siglecs are meant to be a valuable tool to distinguish between self-associated molecular patterns (SAMPs) and pathogen-associated molecular patterns (PAMPs), several pathogens are already known to elude the immune system by mimicking host sialylation and, therefore, masking themselves as SAMPs.

This review is therefore focusing on the role of glycoconjugates of endogenous cells in controlling NET releases and impairing the negative outcome of NETs as well as on the role of glycoconjugates of pathogens that influence NET formation or that directly influence NET release and the biological activity of NETs.

\section{THE INTERACTION BETWEEN GLYCANS AND NEUTROPHILS: A PHYSIOLOGICAL CONTROL SYSTEM IN CIRCULATION}

An impaired NET release and missing NET clearance are, for instance, associated with the formation of a thrombus (7, 40, 41). Not only do the NET fibers serve as a scaffold for the formation of a thrombus. Furthermore, neutrophil elastase is released in the extracellular area and inhibits among others the anticoagulants antithrombin and tissue factor pathway inhibitor. In addition, extracellular histones are known to increase the thrombin generation, causing platelet activation and coagulation (40-43). Therefore, it is not surprising that an impaired NET release within circulation needs to be prevented.

Lizcano et al. investigated the reason why isolated neutrophils are more susceptible to undergoing NETosis than neutrophils within circulation (44). They determined that glycophorin A, a sialoglycoprotein that is located on the surface of erythrocytes, is a candidate that might be responsible for this effect. It is able to bind Siglec-9 on the surface of neutrophils, inhibiting neutrophil activation within circulation (Figure 1A). Remarkably, modification of the sialic acid on the surface of the erythrocytes prevents the outlined inhibitory effects (44). Interestingly, also cancer cells, which are characterized by hypersialylation, seem to use Siglec-5 and Siglec-9 to evade entrapment by activated neutrophils [excellently reviewed by Adams et al. (45) and Rodrigues and Macauley (46)].

Besides sialylated glycoconjugates, heparin, a well-known anticoagulant, inhibits neutrophil degranulation and aggregation in vitro (47). Furthermore, the influence of low molecular weight heparin, unfractionated heparin, O-desulfated heparin, hyaluronic acid, dextran sulfate, and poly-L-glutamic acid on neutrophil activation was investigated (48). The activation of neutrophils with different stimuli induced the release of neutrophil elastase. However, the application of the different stimuli in combination with low molecular weight heparin as well as dextran sulfate inhibited neutrophil activation and, therefore, the release of neutrophil elastase (Figure 1A) (48, 49). In contrast, the non-sulfated dextran and poly-L-glutamic acid showed no effect on neutrophil activation, leading to the assumption of a sulfate-dependent process. Furthermore, $\mathrm{Xu}$ et al. investigated the role of heparan sulfate in the biology of NETs (50). In heparan sulfate uronyl 2-O-sulgotransferase deficient mice less NET is formed after stimulation with group B streptococcus (GBS). Remarkably, when NET was treated with heparan lyase, its antimicrobial activity decreased (50). Thus, the formation and the activity of NETs seem to be modulated by heparan sulfate.

Besides heparan sulfate, other glycans are known to influence the biological activity of NETs. In this context, Brown et al. focused on neutrophil elastase and neutrophil-induced human bronchial epithelia cell detachment (48). Whilst hyaluronic acid had no effect, low-molecular-weight heparin, unfractionated heparin, O-desulfated heparin, and dextran sulfate significantly inhibited the neutrophil elastase-induced detachment (48). Furthermore, Fuchs et al. published that the treatment of NETs with heparin destroys their scaffold and prevents the formation of a thrombus (Figure 1B). Heparin has a high-charge-dependent affinity to histones $(51,52)$ and is able to release histones from chromatin fibers, therefore, destabilizing NETs (Figure 1B) (40). Since histones that are released during NETs are able to damage negatively charged cell membranes, histones are often described as antimicrobial peptides (AMPs) that are released during NETs alongside with other antimicrobial biomolecules like lactoferrin 


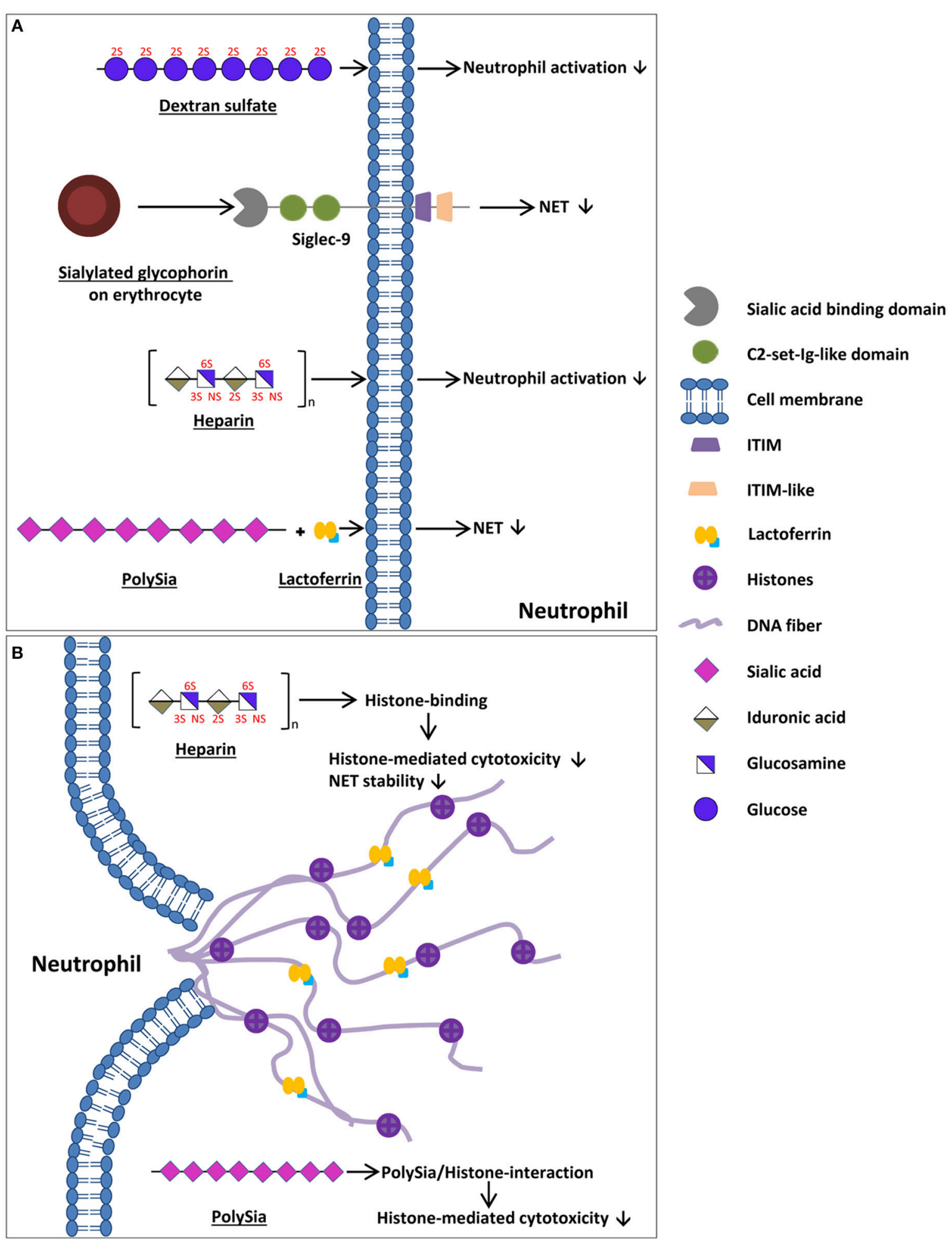

FIGURE 1 | Mechanisms of endogenous cells and naturally occurring components to enhance bacterial entrapment, to decrease impaired NET release, and to decrease the cytotoxic outcome of NETs. (A) Natural occurring components preventing NET release/neutrophil degranulation. Glycophorin is a sialoglycoprotein located at the surface of erythrocytes that inhibits NET release/neutrophil activation via sialic acid binding to Siglec-9 within circulation. Furthermore, the GAG heparin as well as dextran sulfate inhibit neutrophil activation in a sulfate-dependent manner. The interaction of lactoferrin with polySia increases the inhibition of NET releases. (B) Mechanisms to decrease the cytotoxic outcome of NETs for a body's own cells as well as to increase bacterial entrapment. PolySia as well as GAGs, such as heparin, bind to released histones, reducing histone-mediated cytotoxicity. The cartoons of all polysaccharides show only exemplary parts of these polymers and may differ from the actual structure (e.g., chain length and composition). 
and neutrophil elastase $(1,6,53-56)$. Unfortunately, these properties of all histones ( $\mathrm{H} 1, \mathrm{H} 2 \mathrm{~A}, \mathrm{H} 2 \mathrm{~B}, \mathrm{H} 3$, and $\mathrm{H} 4)$ are toxic not only for pathogens but also for endogenous cells (5760 ). Within the plasma, the cytotoxicity of histones is reduced by the inter-alpha-inhibitor-protein (IAIP) associated with high molecular weight hyaluronic acid and chondroitin sulfate. IAIP as well as high molecular weight hyaluronic acid and chondroitin sulfate bind recombinant histone $\mathrm{H} 4$, contributing to reduced histone mediated cytotoxicity (Figure 1B) (61).

In addition to GAGs, such as hepain (52), another linear carbohydrate, polysialic acid (polySia), is a naturally occurring inhibitor of the cytotoxic effects of histones $(59,62,63)$. Remarkably, polySia was detected in the plasma of different species, from fish to humankind, and may represent a natural buffer system for the inactivation of the cytotoxicity of extracellular histones in blood (Figure 1B) (64). PolySia influences histone-mediated cytotoxicity in a concentration as well as in a chain-length-dependent manner $(65,66)$. In line with that, polysialylated nanoparticles and in vitro polysialylated cervical mucins represent tools to counteract histone-mediated cytotoxicity during an exaggerated NET formation $(65,66)$. Interestingly, quite recently, Kühnle et al. $(67,68)$ published that polySia interacts with lactoferrin. Lactoferrin is known to inhibit a NET release by forming a "lactoferrin-shell" around the activated neutrophils (69). In vitro experiments suggested that the efficiency of lactoferrin in preventing the release of NETs was enhanced in the presence of polySia (67).

Thus, the presented examples show endogenous glycandependent ways to control the release of NETs in addition to decreasing their damaging effects, indicating, once more, the widespread function of glycosylation within the field of immunology. However, it has to be considered that a medal always has two sides. Some pathogens exploit the previously described mechanisms for their own purposes, as described in the next chapter.

\section{THE GLYCOSYLATION OF PATHOGENS: A POWERFUL TOOL TO ESCAPE NETS}

\section{Bacteria}

During their evolution, several pathogens have "learned" to use carbohydrate-dependent mechanisms to modulate the immune system. For instance, distinct bacteria strains target Siglecs to circumvent the release of NETs by neutrophils. Pseudomonas aeruginosa ( $P$. aeruginosa), for example, can use sialic acids from its hosts to decorate their glycoconjugates with sialic acids. On the surface of $P$. aeruginosa, $N$-acetylneuraminic acid (Neu5Ac), $\mathrm{N}$-glycolylneuraminic acid (Neu5Gc), and 9-Oacetyl- $N$-acetylneuraminic acid (Neu5,9Ac2) have been found, enabling $P$. aeruginosa to inhibit neutrophil activation via the activation of Siglec-9 (70). According to Khatua et al. $P$. aeruginosa directly binds to the neutrophil via Siglec9, stimulating the production of cytokine IL-10 and TGF$\beta$ (71). The generation of ROS is inhibited, and a decrease of the release of neutrophil elastase is detected. Since ROS production can be an initial step of NETosis, it is not surprising that the release of NETs is inhibited by the binding partners of Siglec-9 on the surface of $P$. aeruginosa (Figure 2) $(72,73)$.

However, $P$. aeruginosa is not the only pathogen using Siglec-9 to circumvent the activation of neutrophils. Carlin et al. (74) also showed that GBS is able to inhibit neutrophil activation through Siglec-9. GBS eluded the human immune system, causing invasive infections in human newborns by hosting the common terminus of human glycoproteins, $\operatorname{Sia} \alpha(2,3) \mathrm{Gal} \beta(1,4) \mathrm{GlcNAc}$, on their capsular polysaccharide. Here, the sialic acid binding of GBS to neutrophil Siglec-9 also initiated the production of the NET-suppressive cytokine IL-10 (74).

In addition to these findings, glycans of GBS interact with Siglec-5 and Siglec-14 (75). These Siglecs are an example of an antagonistic interplay between Siglecs. The sialic acid binding of Siglec-14 counteracts the pathogen-induced suppression of neutrophil activation (Figure 2). Intriguingly, the absence of Siglec-14 due to Siglec-14 null-polymorphism in humans leads to the increased susceptibility of neutrophils to GBS. The relevance of this pathogen-induced inhibition of neutrophil activation becomes apparent through the discovery of the Siglec5 and Siglec-14 expressions on amniotic epithelium (75). These epithelial cells are the site of the initial contact area of the fetus and the pathogens. Ali et al. suggested that Siglec-14 nullpolymorphism might relate to the risk of prematurity during GBS invasion.

In 2016, a surprising discovery was made by Secundino et al. (76). While investigating GBS and its capability to bind and activate Siglec-9 via its sialylated glycans, they observed that Siglec-9 also bound high molecular weight hyaluronan, consisting of repeating disaccharide units of $\mathrm{N}$ acetylglucosamine (GlcNAc) and glucuronic acid (GlcA), with alternating $\beta 1,4-$ and $\beta 1,3$-linkages. Intriguingly, they detected a new, specific binding site, apart from the V-set Ig-like domain. Since the capsular polysaccharide of group A streptococcus (GAS) contains high-molecular-weight hyaluronan units, NETs' formation and oxidative bursts were prevented (Figure 2) (76). Remarkably, a single inhibitory Siglec recognizes two different glycan motifs as SAMPs, leading to the suppression of neutrophil activation.

Although GBS seems to be the best studied pathogen regarding sialic acids and NETs inhibition, several more pathogens, like Campylobacter jejuni, Neisseria gonorrhoeae, and Escherichia coli K1, are also able to synthesize sialylated glycans, leading to the assumption that comparable strategies are also used here to escape NETs $(70,77-79)$. Since polySia is also able to modulate NET formation and the activity of NETs, it seems likely that polySia-positive bacteria, like Escherichia coli K1 and distinct Neisseria meningitides strains, are able to trigger polySiadependent mechanisms $(80,81)$. However, until now, no study has examined the impact of polySia during their invasion in the context of NETs.

Interestingly, obstructing NET release is not the only tool of bacteria, to elude the immune system. Several pathogens are able to circumvent NET-mediated killing. Streptococcus pneumoniae (S. pneumoniae) for instance, which is one of the major causes of mortality and morbidity, circumvents NET-mediated 


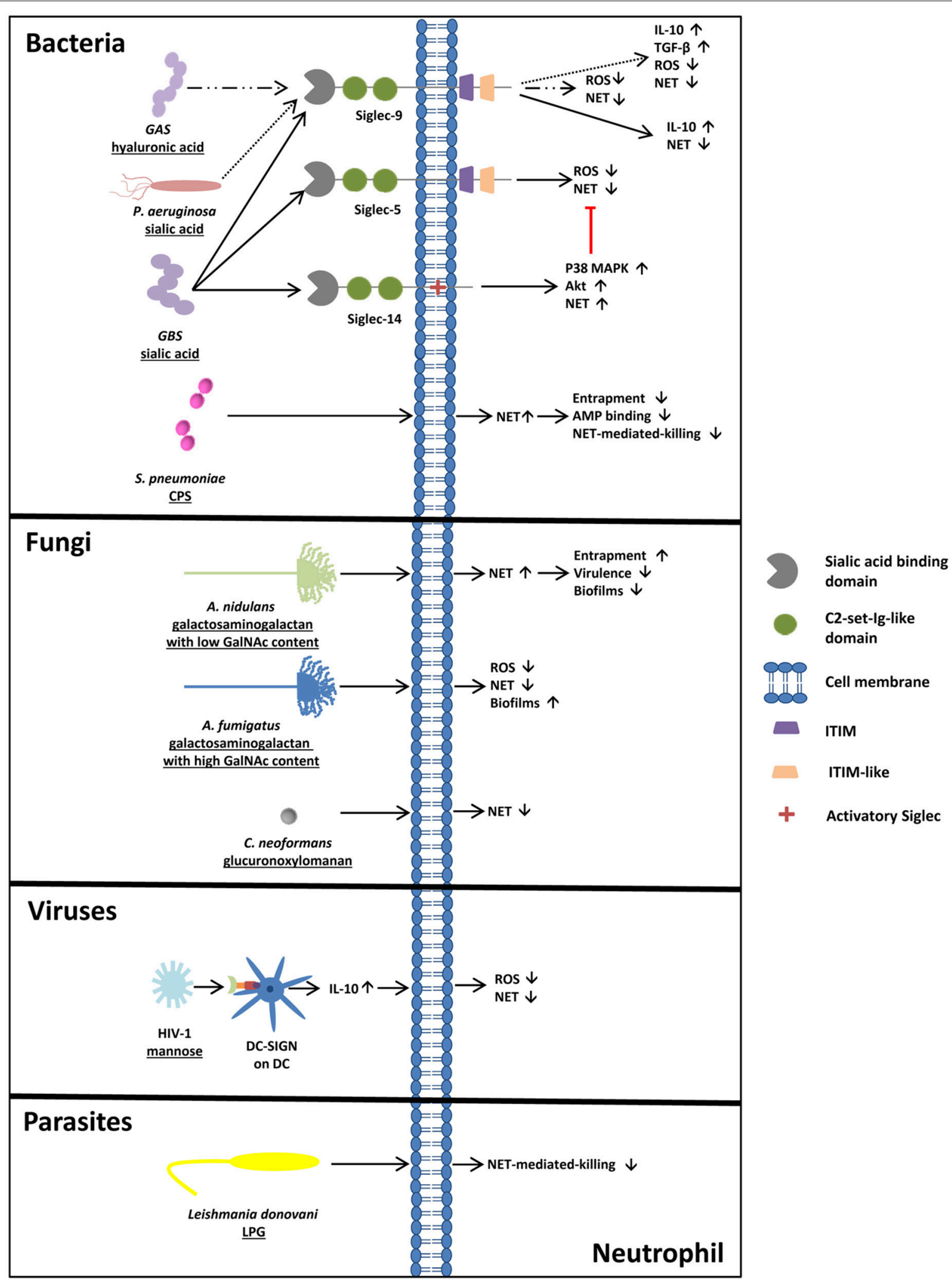

FIGURE 2 | Glycosylation of pathogens- a powerful tool to circumvent NET-mediated entrapment and killing. Bacteria circumventing NET release or NET-mediated killing. S. pneumoniae induces NET release, but NET-mediated killing and entrapment is bypassed due to the capsular polysaccharide (CPS). Pathogens like GBS, 
FIGURE 2 | GAS, and P. aeruginosa exploit Siglecs for their own purposes. Via sialic/hyaluronic acid binding to Siglecs, IL-10 as well as TGF- $\beta$ production are upregulated and NET release is inhibited. Also fungi circumvent NET release/ NET entrapment by glycans. Compared to the less pathogenic $A$. nidulans, surrounded by galactosaminogalactan with a low amount of GalNAc, $A$. fumigatus, surrounded by galactosaminogalactan with a high GalNAc content, inhibits NET release and ROS production and forms more adherent biofilms, explaining the differences in virulence of these two fungi strains. In addition, the capsular strain of $C$. neoformans inhibits the release of NETs due to its glucuronoxylomannan coating, whereas acapsular strains of $C$. neoformans induce NETosis. In addition, sialylated viruses like HIV-1 prevent ROS-dependent NET release by DC-SIGN engagement and parasites, like Leishmania donovani circumvent NET mediated killing by lipopeptidoglycan $(L P G)$ on its surface.

killing through its polysaccharide capsule (82). Encapsulated $S$. pneumoniae strains show significantly reduced trapping by NETs compared to non-capsulated strains (Figure 2). Furthermore, S. pneumoniae contain positively charged lipoteichoic acid on their surface, which increases the electrochemical repulsion of antimicrobial peptides (82).

\section{Fungi}

Moreover, it is not only bacteria that circumvent NETmediated entrapment and killing. In their research, Rocha et al. (83) detected that the fungus Cryptococcus neoformans (C. neoformans) is surrounded by capsular polysaccharides containing glucuronoxylomannan. The wild type of strain inhibits the release of NETs, whereas acapsular mutants or mutants surrounded by glucuronoxylomannogalactan induce NETosis (Figure 2) (83).

Furthermore, Aspergillus fumigatus (A. fumigatus), which accounts up for around $80 \%$ of all invasive Aspergillus infections, shows resistance against NET-induced damage due to the production of cell-wall-associated galactosaminogalactan and a secreted form of galactosaminogalactan. Galactosaminogalactan consists of galactose and N-acetylgalactosamine (GalNAc) residues. Since galactosaminogalactans play a certain role in host-pathogen interactions, as they are required for biofilm formation, a galactosaminogalactan-deficient mutant of $A$. fumigatus exhibited reduced virulence (Figure 2) (84-88).

Interestingly, Aspergillus nidulans (A. nidulans), a strain producing galactosaminogalactans with a lower content of GalNAc residues in comparison to $A$. fumigatus, was found to be less pathogenic, formed less-adherent biofilms, and was, therefore, more susceptible to NET-induced damage. Since A. nidulans is only known to induce pathologies in patients with chronic granulomatous disease (CGD), characterized by an impaired NADPH oxidase complex, further investigations concerning the influence of NADPH oxidase revealed that cell-wall-bound galactosaminogalactans in A. fumigatus enhance resistance against NADPH-oxidase-dependent neutrophil extracellular damage. This might explain the increased virulence of A. nidulans in CGD patients (88).

\section{Viruses}

Interestingly, also viruses are able to evade immune control mechanisms. The HIV-1 virus, for instance, counteracts NET formation by engaging the C-type lectin DC-SIGN (CD209) on dendritic cells via its envelope glycoprotein containing more high mannose than complex $\mathrm{N}$-glycan structures. The binding induces the production of IL-10, contributing to the inhibition of
ROS-dependent NET release upon TLR7 and TLR8 engagement (89) (Figure 2). This study let suggest that also glycans of other viruses can target DC-SIGN, such as Ebola virus, the Japanese encephalitis virus and of the hepatitis $C$ virus, that may also influence in an indirect way the formation of NET (90-92).

\section{Parasites}

Leishmania donovani, a protozoan parasite, contains lipopeptidoglycan on its surface and mutants lacking lipopeptidoglycan show less survival in NET in comparison to the wild type strain (Figure 2) (93). In addition, virulent strains of Leishmania donovani can contain high amount of sialylated glycans representing binding partners for Siglec-5 leading to an inhibition of ROS production in macrophages (94). Comparable mechanism of these Leishmania donovani glycans, which are terminated with $\alpha 2,3-$ and $\alpha 2,6$-linked sialic acid residues, may also take place on neutrophils and might also be used by other sialic acid positive parasites.

\section{CONCLUSION}

This review gives a short summary concerning the impact of glycans to modulate the formation and activity of NET describing glycan dependent mechanisms of endogenous cells to prevent the activation of neutrophils or to inactivate cytotoxic molecules of NET, which are, however, also used as an escape strategy by distinct pathogens. All of the outlined examples show that glycans play a key role in the biology of NET and that they have great potential as a therapeutic tool in NETs-associated pathologies.

\section{AUTHOR CONTRIBUTIONS}

All authors listed have made a substantial, direct and intellectual contribution to the work, and approved it for publication.

\section{FUNDING}

This work was partially financed by a grant of the Deutsche Forschungsgemeinschaft (GA 1755/1-2). The publication of this article was funded by the Open Access Fund of the Leibniz Institute for Farm Animal Biology (FBN).

\section{ACKNOWLEDGMENTS}

We thank our team for critical remarks. 


\section{REFERENCES}

1. Brinkmann V, Reichard U, Goosmann C, Fauler B, Uhlemann Y, Weiss DS, et al. Neutrophil extracellular traps kill bacteria. Science. (2004) 303:1532-5. doi: $10.1126 /$ science. 1092385

2. Byrd AS, O’Brien XM, Johnson CM, Lavigne LM, Reichner JS. An extracellular matrix-based mechanism of rapid neutrophil extracellular trap formation in response to Candida albicans. J Immunol. (2013) 190:4136-48. doi: 10.4049/jimmunol.1202671

3. Brinkmann V, Zychlinsky A. Neutrophil extracellular traps: is immunity the second function of chromatin? J Cell Biol. (2012) 198:773-83. doi: $10.1083 /$ jcb. 201203170

4. Neeli I, Radic M. Opposition between PKC isoforms regulates histone deimination and neutrophil extracellular chromatin release. Front Immunol. (2013) 4:38. doi: 10.3389/fimmu.2013.00038

5. Boeltz S, Amini P, Anders HJ, Andrade F, Bilyy R, Chatfield S, et al. To NET or not to NET:current opinions and state of the science regarding the formation of neutrophil extracellular traps. Cell Death Differ. (2019) 26:395-408. doi: 10.1038/s41418-018-0261-x

6. Papayannopoulos V, Zychlinsky A. NETs: a new strategy for using old weapons. Trends Immunol. (2009) 30:513-21. doi: 10.1016/j.it.2009.07.011

7. Brill A, Fuchs TA, Savchenko AS, Thomas GM, Martinod K, De Meyer SF, et al. Neutrophil extracellular traps promote deep vein thrombosis in mice. J Thromb Haemost. (2012) 10:136-44. doi: 10.1111/j.1538-7836.2011. 04544.x

8. Andrews RK, Arthur JF, Gardiner EE. Neutrophil extracellular traps (NETs) and the role of platelets in infection. Thromb Haemost. (2014) 112:659-65. doi: 10.1160/TH14-05-0455

9. Rahman S, Gadjeva M. Does NETosis contribute to the bacterial pathoadaptation in cystic fibrosis? Front Immunol. (2014) 5:378. doi: 10.3389/fimmu.2014.00378

10. Gray RD, McCullagh BN, McCray PB. NETs and CF lung disease: current status and future prospects. Antibiotics. (2015) 4:62-75. doi: 10.3390/antibiotics4010062

11. Pruchniak MP, Kotula I, Manda-Handzlik A. Neutrophil extracellular traps (Nets) impact upon autoimmune disorders. Cent Eur J Immunol. (2015) 40:217-24. doi: 10.5114/ceji.2015.52836

12. Stephan A, Fabri M. The NET, the trap and the pathogen: neutrophil extracellular traps in cutaneous immunity. Exp Dermatol. (2015) 24:161-6. doi: 10.1111/exd.12599

13. Giaglis S, Hahn S, Hasler P. The NET outcome: are neutrophil extracellular traps of any relevance to the pathophysiology of autoimmune disorders in childhood? Front Pediatr. (2016) 4:97. doi: 10.3389/fped.2016.00097

14. Hoffmann JH, Enk AH. Neutrophil extracellular traps in dermatology: caught in the NET. J Dermatol Sci. (2016) 84:3-10. doi: 10.1016/j.jdermsci.2016.07.001

15. Brinkmann V. Neutrophil extracellular traps in the second decade. J Innate Immun. (2018) 10:414-21. doi: 10.1159/000489829

16. Cedervall J, Hamidi A, Olsson AK. Platelets, NETs and cancer. Thromb Res. (2018) 164(Suppl 1):S148-52. doi: 10.1016/j.thromres.2018.01.049

17. Hart GW, Copeland RJ. Glycomics hits the big time. Cell. (2010) 143:672-6. doi: 10.1016/j.cell.2010.11.008

18. Macauley MS, Crocker PR, Paulson JC. Siglec-mediated regulation of immune cell function in disease. Nat Rev Immunol. (2014) 14:653-66. doi: $10.1038 /$ nri3737

19. Varki A. Biological roles of glycans. Glycobiology. (2017) 27:3-49. doi: 10.1093/glycob/cww086

20. Gagneux P, Aebi M, Varki A. Evolution of glycan diversity. In: Varki A, Cummings RD, Esko JD, Stanley P, Hart GW, Aebi M, Darvill AG, Kinoshita T, Packer NH, Prestegard JH, Schnaar Rl, Seeberger PH, editors. Essentials of Glycobiology. Cold Spring Harbor, NY: The Consortium of Glycobiology (2015). p. 253-64.

21. Lepenies B, Yin J, Seeberger PH. Applications of synthetic carbohydrates to chemical biology. Curr Opin Chem Biol. (2010) 14:404-11. doi: 10.1016/j.cbpa.2010.02.016

22. Zhou JY, Oswald DM, Oliva KD, Kreisman LSC, Cobb BA. The glycoscience of immunity. Trends Immunol. (2018) 39:523-35. doi: 10.1016/j.it.2018. 04.004
23. Gandhi NS, Mancera RL. The structure of glycosaminoglycans and their interactions with proteins. Chem Biol Drug Des. (2008) 72:455-82. doi: 10.1111/j.1747-0285.2008.00741.x

24. Iozzo RV, San Antonio JD. Heparan sulfate proteoglycans: heavy hitters in the angiogenesis arena. J Clin Investig. (2001) 108:349-55. doi: 10.1172/JCI13738

25. Sanderson RD. Heparan sulfate proteoglycans in invasion and metastasis. Semin Cell Dev Biol. (2001) 12:89-98. doi: 10.1006/scdb.2000.0241

26. Liu D, Shriver Z, Qi Y, Venkataraman G, Sasisekharan R. Dynamic regulation of tumor growth and metastasis by heparan sulfate glycosaminoglycans. Semin Thromb Hemost. (2002) 28:67-78. doi: 10.1055/s-2002-20565

27. Fareed J, Hoppensteadt DA, Bick RL. An update on heparins at the beginning of the new millennium. Semin Thromb Hemost. (2000) 26:005-22. doi: 10.1055/s-2000-9498

28. Benito C, Marco G, Giangiacomo T. Structural and conformational aspects of the anticoagulant and antithrombotic activity of heparin and dermatan sulfate. Curr Pharmaceut Design. (2004) 10:939-49. doi: 10.2174/1381612043452794

29. Schauer R. Sialic acids: fascinating sugars in higher animals and man. Zoology. (2004) 107:49-64. doi: 10.1016/j.zool.2003.10.002

30. Schauer R. Sialic acids as regulators of molecular and cellular interactions. Curr Opin Struct Biol. (2009) 19:507-14. doi: 10.1016/j.sbi.2009.06.003

31. Colley KJ, Kitajima K, Sato C. Polysialic acid: biosynthesis, novel functions and applications. Crit Rev Biochem Mol Biol. (2014) 49:498-532. doi: $10.3109 / 10409238.2014 .976606$

32. Schnaar RL, Gerardy-Schahn R, Hildebrandt H. Sialic acids in the brain: gangliosides and polysialic Acid in nervous system development, stability, disease, and regeneration. Physiol Rev. (2014) 94:461-518. doi: 10.1152/physrev.00033.2013

33. Galuska CE, Lutteke T, Galuska SP. Is polysialylated NCAM not only a regulator during brain development but also during the formation of other organs? Biology. (2017) 6:1-15. doi: 10.3390/biology6020027

34. Crocker PR. Siglecs in innate immunity. Curr Opin Pharmacol. (2005) 5:4317. doi: 10.1016/j.coph.2005.03.003

35. Varki A, Angata T. Siglecs-the major subfamily of I-type lectins. Glycobiology. (2006) 16:1R-27R. doi: 10.1093/glycob/cwj008

36. Crocker PR, Paulson JC, Varki A. Siglecs and their roles in the immune system. Nat Rev Immunol. (2007) 7:255-66. doi: 10.1038/nri2056

37. Bochner BS, Zimmermann N. Role of Siglecs and related glycan-binding proteins in immune responses and immunoregulation. J Allergy Clin Immunol. (2015). 135:598-608. doi: 10.1016/j.jaci.2014.11.031

38. Angata T. Possible influences of endogenous and exogenous ligands on the evolution of human Siglecs. Front Immunol. (2018) 9:2885. doi: 10.3389/fimmu.2018.02885

39. Bornhofft KF, Goldammer T, Rebl A, Galuska SP. Siglecs: a journey through the evolution of sialic acid-binding immunoglobulin-type lectins. Dev Comp Immunol. (2018) 86:219-31. doi: 10.1016/j.dci.2018.05.008

40. Fuchs TA, Brill A, Duerschmied D, Schatzberg D, Monestier M, Myers DD Jr, et al. Extracellular DNA traps promote thrombosis. Proc Natl Acad Sci USA. (2010) 107:15880-5. doi: 10.1073/pnas.1005743107

41. von Brühl M-L, Stark K, Steinhart A, Chandraratne S, Konrad I, Lorenz $\mathrm{M}$, et al. Monocytes, neutrophils, and platelets cooperate to initiate and propagate venous thrombosis in mice in vivo. J Exp Med. (2012) 209:819. doi: $10.1084 /$ jem. 20112322

42. Clark SR, Ma AC, Tavener SA, McDonald B, Goodarzi Z, Kelly MM, et al. Platelet TLR4 activates neutrophil extracellular traps to ensnare bacteria in septic blood. Nat Med. (2007) 13:463. doi: 10.1038/nm1565

43. Caudrillier A, Kessenbrock K, Gilliss BM, Nguyen JX, Marques MB, Monestier $\mathrm{M}$, et al. Platelets induce neutrophil extracellular traps in transfusion-related acute lung injury. J Clin Invest. (2012) 122:2661-71. doi: 10.1172/JCI61303

44. Lizcano A, Secundino I, Dohrmann S, Corriden R, Rohena C, Diaz S, et al. Erythrocyte sialoglycoproteins engage Siglec-9 on neutrophils to suppress activation. Blood. (2017) 129:3100-10. doi: 10.1182/blood-2016-11-751636

45. Adams OJ, Stanczak MA, von Gunten S, Laubli H. Targeting sialic acid-Siglec interactions to reverse immune suppression in cancer. Glycobiology. (2018) 28:640-7. doi: 10.1093/glycob/cwx108

46. Rodrigues E, Macauley MS. Hypersialylation in cancer: modulation of inflammation and therapeutic opportunities. Cancers. (2018) 10:207. doi: $10.3390 /$ cancers 10060207 
47. Brown RA, Lever R, Jones NA, Page CP. Effects of heparin and related molecules upon neutrophil aggregation and elastase release in vitro. $\mathrm{Br} J$ Pharmacol. (2003) 139:845-53. doi: 10.1038/sj.bjp.0705291

48. Brown RA, Leung E, Kankaanranta H, Moilanen E, Page CP. Effects of heparin and related drugs on neutrophil function. Pulmon Pharmacol Therapeut. (2012) 25:185-92. doi: 10.1016/j.pupt.2012.01.006

49. El Habbal MH, Smith L, Elliott MJ, Strobel S. Effect of heparin anticoagulation on neutrophil adhesion molecules and release of IL8: C3 is not essential. Cardiovasc Res. (1995) 30:676-81. doi: 10.1016/S0008-6363(95)00069-0

50. Xu D, Olson J, Cole JN, van Wijk XM, Brinkmann V, Zychlinsky A, et al. Heparan sulfate modulates neutrophil and endothelial function in antibacterial innate immunity. Infect Immun. (2015) 83:3648-56. doi: 10.1128/IAI.00545-15

51. Pal PK, Starr T, Gertler MM. Neutralization of heparin by histone and its subfractions. Thromb Res. (1983) 31:69-79. doi: 10.1016/0049-3848(83)90008-7

52. Wildhagen KC, Garcia de Frutos P, Reutelingsperger CP, Schrijver R, Areste C, Ortega-Gomez A, et al. Nonanticoagulant heparin prevents histone-mediated cytotoxicity in vitro and improves survival in sepsis. Blood. (2014) 123:1098101. doi: 10.1182/blood-2013-07-514984

53. Miller BF, Abrams R, Dorfman A, Klein M. Antibacterial properties of protamine and histone. Science. (1942) 96:428-30. doi: $10.1126 /$ science.96.2497.428

54. Nauseef WM. How human neutrophils kill and degrade microbes: an integrated view. Immunol Rev. (2007) 219:88-102. doi: 10.1111/j.1600-065X.2007.00550.x

55. Kawasaki H, Iwamuro S. Potential roles of histones in host defense as antimicrobial agents. Infect Disord Drug Targets. (2008) 8:195-205. doi: 10.2174/1871526510808030195

56. Urban CF, Ermert D, Schmid M, Abu-Abed U, Goosmann C, Nacken W, et al. Neutrophil extracellular traps contain calprotectin, a cytosolic protein complex involved in host defense against Candida albicans. PLoS Pathog. (2009) 5:e1000639. doi: 10.1371/journal.ppat.1000639

57. Hariton-Gazal E, Rosenbluh J, Graessmann A, Gilon C, Loyter A. Direct translocation of histone molecules across cell membranes. J Cell Sci. (2003) 116(Pt 22):4577-86. doi: 10.1242/jcs.00757

58. Rosenbluh J, Singh SK, Gafni Y, Graessmann A, Loyter A. Nonendocytic penetration of core histones into petunia protoplasts and cultured cells: a novel mechanism for the introduction of macromolecules into plant cells. Biochim Biophys Acta. (2004) 1664:230-40. doi: 10.1016/j.bbamem.2004.06.003

59. Zlatina K, Lutteke T, Galuska SP. Individual impact of distinct polysialic acid chain lengths on the cytotoxicity of histone $\mathrm{H} 1, \mathrm{H} 2 \mathrm{~A}, \mathrm{H} 2 \mathrm{~B}, \mathrm{H} 3$ and $\mathrm{H} 4$. Polymers. (2017) 9:720. doi: 10.3390/polym9120720

60. Zlatina K, Galuska SP. Polysialic acid modulates only the antimicrobial properties of distinct histones. ACS Omega. (2019) 4:1601-10. doi: 10.1021/acsomega.8b02222

61. Chaaban H, Keshari RS, Silasi-Mansat R, Popescu NI, Mehta D, Souza P, et al. Inter-alpha inhibitor protein and its associated glycosaminoglycans protect against histone-induced injury. (2015). 125:2286-96. doi: 10.1182/blood-2014-06-582759

62. Saffarzadeh M, Juenemann C, Queisser MA, Lochnit G, Barreto G, Galuska SP, et al. Neutrophil extracellular traps directly induce epithelial and endothelial cell death: a predominant role of histones. PLoS ONE. (2012) 7:e32366. doi: 10.1371/journal.pone.0032366

63. Ulm C, Saffarzadeh M, Mahavadi P, Müller S, Prem G, Saboor F, et al. Soluble polysialylated NCAM: a novel player of the innate immune system in the lung. Cell Mol Life Sci. (2013) 70:3695-708. doi: 10.1007/s00018-013-1342-0

64. Zlatina K, Saftenberger M, Kuhnle A, Galuska CE, Gartner U, Rebl A, et al. Polysialic acid in human plasma can compensate the cytotoxicity of histones. Int J Mol Sci. (2018) 19:1-10. doi: 10.3390/ijms19061679

65. Galuska CE, Dambon JA, Kuhnle A, Bornhofft KF, Prem G, Zlatina K, et al. Artificial polysialic acid chains as sialidase-resistant molecular-anchors to accumulate particles on neutrophil extracellular traps. Front Immunol. (2017) 8:1229. doi: 10.3389/fimmu.2017.01229

66. Galuska SP, Galuska CE, Tharmalingam T, Zlatina K, Prem G, Husejnov FCO, et al. In vitro generation of polysialylated cervical mucins by bacterial polysialyltransferases to counteract cytotoxicity of extracellular histones. FEBS J. (2017) 284:1688-99. doi: 10.1111/febs 14073

67. Kühnle A, Veelken R, Galuska CE, Saftenberger M, Verleih MH, Galuska SP. Polysialic acid interacts with lactoferrin and supports its activity to inhibit the release of neutrophil extracellular traps. Carbohydr Polym. (2019) 208:32-41. doi: 10.1016/j.carbpol.2018.12.033

68. Kuhnle A, Lutteke T, Bornhofft KF, Galuska SP. Polysialic acid modulates the binding of external lactoferrin in neutrophil extracellular traps. Biology. (2019) 8:20. doi: 10.3390/biology8020020

69. Okubo K, Kamiya M, Urano Y, Nishi H, Herter JM, Mayadas T, et al. Lactoferrin suppresses neutrophil extracellular traps release in inflammation. EBioMedicine. (2016) 10:204-15. doi: 10.1016/j.ebiom.2016.07.012

70. Khatua B, Roy S, Mandal C. Sialic acids siglec interaction: a unique strategy to circumvent innate immune response by pathogens. Ind J Med Res. (2013) 138:648-62.

71. Khatua B, Bhattacharya K, Mandal C. Sialoglycoproteins adsorbed by Pseudomonas aeruginosa facilitate their survival by impeding neutrophil extracellular trap through siglec-9. J Leukoc Biol. (2012) 9:641-55. doi: 10.1189/jlb.0511260

72. Hahn S, Giaglis S, Chowdhury CS, Hosli I, Hasler P. Modulation of neutrophil NETosis: interplay between infectious agents and underlying host physiology. Semin Immunopathol. (2013) 35:439-53. doi: 10.1007/s00281-013-0380-x

73. Fuchs TA, Abed U, Goosmann C, Hurwitz R, Schulze I, Wahn V, et al. Novel cell death program leads to neutrophil extracellular traps. J Cell Biol. (2007) 176:231. doi: $10.1083 /$ jcb.200606027

74. Carlin AF, Uchiyama S, Chang YC, Lewis AL, Nizet V, Varki A. Molecular mimicry of host sialylated glycans allows a bacterial pathogen to engage neutrophil Siglec-9 and dampen the innate immune response. Blood. (2009) 113:3333-6. doi: 10.1182/blood-2008-11-187302

75. Ali SR, Fong JJ, Carlin AF, Busch TD, Linden R, Angata T, et al. Siglec5 and Siglec-14 are polymorphic paired receptors that modulate neutrophil and amnion signaling responses to group B Streptococcus. J Exp Med. (2014) 211:1231-42. doi: 10.1084/jem.20131853

76. Secundino I, Lizcano A, Roupe KM, Wang X, Cole JN, Olson J, et al. Host and pathogen hyaluronan signal through human siglec-9 to suppress neutrophil activation. J Mol Med. (2016) 94:219-33. doi: 10.1007/s00109-015-1341-8

77. Vimr E, Lichtensteiger C. To sialylate, or not to sialylate: that is the question. Trends Microbiol. (2002) 10:254-7. doi: 10.1016/S0966-842X(02)02361-2

78. Schwarz F, Landig CS, Siddiqui S, Secundino I, Olson J, Varki N, et al. Paired Siglec receptors generate opposite inflammatory responses to a humanspecific pathogen. EMBO J. (2017) 36:751-60. doi: 10.15252/embj.2016 95581

79. Landig CS, Hazel A, Kellman BP, Fong JJ, Schwarz F, Agarwal S, et al. Evolution of the exclusively human pathogen Neisseria gonorrhoeae: humanspecific engagement of immunoregulatory Siglecs. Evol Appl. (2019) 12:33749. doi: 10.1111/eva.12744

80. Bhattacharjee AK, Jennings HJ, Kenny CP, Martin A, Smith IC. Structural determination of the sialic acid polysaccharide antigens of Neisseria meningitidis serogroups B and C with carbon 13 nuclear magnetic resonance. J Biol Chem. (1975) 250:1926-32.

81. Orskov F, Orskov I, Sutton A, Schneerson R, Lin W, Egan W, et al. Form variation in Escherichia coli K1: determined by O-acetylation of the capsular polysaccharide. J Exp Med. (1979) 149:669-85. doi: 10.1084/jem.149.3.669

82. Wartha F, Beiter K, Albiger B, Fernebro J, Zychlinsky A, Normark S, et al. Capsule and D-alanylated lipoteichoic acids protect Streptococcus pneumoniae against neutrophil extracellular traps. Cell Microbiol. (2007) 9:1162-71. doi: 10.1111/j.1462-5822.2006.00857.x

83. Rocha JDB, Nascimento MTC, Decote-Ricardo D, Côrte-Real S, Morrot A, Heise N, et al. Capsular polysaccharides from Cryptococcus neoformans modulate production of neutrophil extracellular traps (NETs) by human neutrophils. Sci Rep. (2015) 5:8008. doi: 10.1038/srep08008

84. Geiser DM, Klich MA, Frisvad JC, Peterson SW, Varga J, Samson R, et al. The current status of species recognition and identification in Aspergillus. Stud Mycol. (2007) 59:1-10. doi: 10.3114/sim.2007.59.01

85. Gravelat FN, Ejzykowicz DE, Chiang LY, Chabot JC, Urb M, Macdonald $\mathrm{KD}$, et al. Aspergillus fumigatus MedA governs adherence, host cell interactions and virulence. Cell Microbiol. (2010) 12:473-88. doi: $10.1111 /$ j.1462-5822.2009.01408.x 
86. Fontaine T, Delangle A, Simenel C, Coddeville B, van Vliet SJ, van Kooyk Y, et al. Galactosaminogalactan, a new immunosuppressive polysaccharide of Aspergillus fumigatus. PLoS Pathog. (2011) 7:e1002372. doi: 10.1371/journal.ppat.1002372

87. Gravelat FN, Beauvais A, Liu H, Lee MJ, Snarr BD, Chen D, et al. Aspergillus galactosaminogalactan mediates adherence to host constituents and conceals hyphal beta-glucan from the immune system. PLoS Pathog. (2013) 9:e1003575. doi: 10.1371/journal.ppat.1003575

88. Lee MJ, Liu H, Barker BM, Snarr BD, Gravelat FN, Al Abdallah Q, et al. The fungal exopolysaccharide galactosaminogalactan mediates virulence by enhancing resistance to neutrophil extracellular traps. PLOS Pathog. (2015) 11:e1005187. doi: 10.1371/journal.ppat.1005187

89. Saitoh T, Komano J, Saitoh Y, Misawa T, Takahama M, Kozaki T, et al. Neutrophil extracellular traps mediate a host defense response to human immunodeficiency virus-1. Cell Host Microbe. (2012) 12:109-16. doi: 10.1016/j.chom.2012.05.015

90. Lin G, Simmons G, Pohlmann S, Baribaud F, Ni H, Leslie GJ, et al. Differential $\mathrm{N}$-linked glycosylation of human immunodeficiency virus and Ebola virus envelope glycoproteins modulates interactions with DC-SIGN and DCSIGNR. J Virol. (2003) 77:1337-46. doi: 10.1128/JVI.77.2.1337-1346.2003

91. Lozach PY, Lortat-Jacob HA, de Lacroix de Lavalette, SI, Foung S, Amara A, et al. DC-SIGN and L-SIGN are high affinity binding receptors for hepatitis C virus glycoprotein E2. J Biol Chem. (2003) 278:20358-66. doi: $10.1074 /$ jbc.M301284200
92. Wang $\mathrm{P}, \mathrm{Hu} \mathrm{K}$, Luo S, Zhang $\mathrm{M}$, Deng $\mathrm{X}$, Li C, et al. DC-SIGN as an attachment factor mediates Japanese encephalitis virus infection of human dendritic cells via interaction with a single high-mannose residue of viral E glycoprotein. Virology. (2016) 488:108-19. doi: 10.1016/j.virol.2015. 11.006

93. Gabriel C, McMaster WR, Girard D, Descoteaux A. Leishmania donovani promastigotes evade the antimicrobial activity of neutrophil extracellular traps. J Immunol. (2010) 185:4319-27. doi: 10.4049/jimmunol. 1000893

94. Roy S, Mandal C. Leishmania donovani utilize sialic acids for binding and phagocytosis in the macrophages through selective utilization of Siglecs and impair the innate immune arm. PLoS Negl Trop Dis. (2016) 10:e0004904. doi: 10.1371/journal.pntd.0004904

Conflict of Interest Statement: The authors declare that the research was conducted in the absence of any commercial or financial relationships that could be construed as a potential conflict of interest.

Copyright (c) 2019 Bornhöft and Galuska. This is an open-access article distributed under the terms of the Creative Commons Attribution License (CC BY). The use, distribution or reproduction in other forums is permitted, provided the original author(s) and the copyright owner(s) are credited and that the original publication in this journal is cited, in accordance with accepted academic practice. No use, distribution or reproduction is permitted which does not comply with these terms. 